\title{
Determination of Individual Mesio-distal Sizes of Front Teeth Upper and Lower Jaw
}

\author{
${ }^{1}$ Olga P. Ivanova, ${ }^{2}$ Sergey V. Chernenko, ${ }^{3}$ Maria V. Vologina, ${ }^{4}$ Viktor I. Shemonaev, ${ }^{5}$ Elena A. Ogonyan \\ $1,3,4,5$ FGBEI VD «The Volgograd State Medical University» of the Ministry of Health of the Russian Federation, Department \\ of Orthodontics \\ ${ }^{2}$ State Institute Post Graduate Education - Branch of Russian Medical Academy of Continuing Vocational Education, \\ Novokuznetsk \\ Email: olgaa-75@mail.ru,orto-premier@yandex.ru
}

Received: $17^{\text {th }}$ July 2019, Accepted: 10 ${ }^{\text {th }}$ August 2019, Published: $31^{\text {st }}$ August 2019

\begin{abstract}
This article focuses on a problem of measuring front teeth's individual size parameters. Teeth size may determine the set of treatment in orthopaedic stomatology, therapeutic stomatology and orthodontics. Choosing the size of artificial teeth is an important phase of denture, since it determines the function and aesthetics of dental prosthesis. In the practice of orthodontics teeth's size parameters allow for dentist to detect the normodontism, microdontism or macrodontism in each specific case. Knowing about tooth crown's sizes allows dentist to model anatomically correct and aesthetic tooth restoration. Authors review different methods of tooth size measuring and point out it's pros and cons. The review highlighted the relationship between craniofacial parameters and the front teeth's width. The results obtained allow dentist to calculate individual mesio-distal parameters of canine, lateral incisor and central incisor in every case.
\end{abstract}

\section{Keywords}

Dimensions of Teeth, Craniometry, Front Teeth, Craniofacial Complex

\section{Introduction}

The specifics of the structure of dentoalveolar arches and the interrelation of their shape with the dimensions of the teeth and the parameters of the craniofacial complex oblige the dentist to master the research methods of odontometry and odontoscopy $[1,2]$. Methods for determining the size of teeth, which have found application in dental practice, along with the advantages have certain disadvantages. For example, orthopedic dentistry uses the Lee method, which consists in determining the width of the front teeth. According to him, the distance from the middle of the right canine to the middle of the left canine of the upper jaw coincides with the width of the base of the nose [3]. This method allows to determine the distance between the canines of the upper jaw, but not the mesio-distal dimensions of each front tooth. Moyers proposes to evaluate the size of permanent canines and premolars of both the upper and lower jaws using the total width of the lower incisors. The lower incisors are selected as a reference because of the low variability of their size, compared with the upper incisors. This method does not require X-rays and can be used for both the upper and lower dentition [4], but in the absence of lower incisors, this method is not applicable.

Tanaka and Johnston are also use the total width of the lower incisors in their method. According to this method, it is necessary to add $10.5 \mathrm{~mm}$ to the half of the mesio-distal width of the four lower incisors to obtain the calculated total width of the canine and mandibular premolars in one quadrant. The dentist can calculate the total width of the canine and premolar of the upper jaw in one quadrant if he adds $11 \mathrm{~mm}$ to the half of the mesio-distal width of the four lower incisors. This method is highly accurate, despite a small systematic error in the direction of overvaluation of the teeth size, and is also convenient because its use requires only a knowledge of the formula from the doctor, without using of any tables or radiographs [4,5], but it has the same disadvantage as the Moyers method.

Stanley and Kerber have developed a graph that allows to calculate the width of the canines directly on the sum of the width of the incisors and premolars. This method has limitations in its use, since it is applicable only to the teeth of the lower jaw [4].

Tonn states that the relationship between the size of the permanent incisors of the upper and lower jaws (Tonn index) is 1.33 [6]. However, this method can only determine the size of the incisors of the upper and lower jaw, without considering the size of the canines.

There are evidences about the correlation between the sum of the mesio-distal diameters of crowns of the four incisors of the upper jaw and the distance between the zygomatic points $Z y-Z y$ in the works of lots of researchers. When the percentage ratio of the sum of the diameters of the four incisors of the upper jaw to the zygomatic diameter Zy-Zy is between $22 \%$ and $25 \%$, the authors propose to consider that the teeth are of medium size (mesodontism) [9]. But this approach does not consider the size of the canines, as well as does not allow to calculate the size of each tooth separately. According to the method of Bolton, the ratio of the mesio-distal dimensions of the teeth of the frontal group (from canine to canine) is determined, obtaining the anterior ratio, which normally constitutes $77.2 \%$ [6,7]. There is a variation of this technique, which consists in comparing the widths of the teeth of each patient with tabular values. Despite all its diagnostic value, the Bolton method does not allow the dentist to calculate the values of dimensions of each teeth in the case of adentia. 
The purpose of our study is to determine the individual sizes of each front tooth (canine, lateral incisor and central incisor) of the upper and lower jaws.

\section{Materials and Methods}

We have measured the parameters of the craniofacial complex and studied the diagnostic plaster models of the jaws of 79 patients of the first period of mature age, who had physiological occlusion during mesodontism.

During an anthropometric study, the distance between the most prominent outward points of the zygomatic arch $\mathrm{Zy}$ (zygion) was measured.

The biometric study of gypsum models consisted in determining the actual mesio-distal width of each of the three front teeth (canine, central and lateral incisor) of the upper and lower jaws. The crown part of the tooth was measured at the widest points: at the level of the cutting edge at the lower incisors and at the level of the equator for all other teeth. The indexes of the correspondence of the sizes of the teeth were determined by the ratio of the mesio-distal sizes of the crowns of the teeth to the interscale distance $(Z y-Z y)$.

\section{Results and Discussion}

After calculations, it was found that:

- the ratio of the distance between the most prominent points of the Zy-Zy zygomatic arches to the sum of the mesiodistal width of the three front teeth (canine, lateral and central incisors) for the upper jaw is $6,0 \pm 1,16$, for the lower jaw is $7,7 \pm 1,12(\mathrm{mM}= \pm 0,13)$;

- the ratio of the distance between the most prominent points of the Zy-Zy zygomatic arches to the sum of the mesiodistal sizes of six front teeth is $3,0 \pm 2,32$ for the upper jaw and 3,85 $\pm 2,24$ for the lower jaw $(\mathrm{mM}= \pm 0,26)$;

- the ratio of the sum of the mesio-distal sizes of the three front teeth to the width of the canine is $3,0 \pm 0,1$, for the upper jaw, and is $2,7 \pm 0,1$ for the lower jaw $(\mathrm{mM}= \pm 0,01)$;

- $\quad$ the ratio of the sum of the mesio-distal sizes of three front teeth to the width of the lateral incisor is $3,4 \pm 0,2$ for the upper jaw, and is $3,0 \pm 0,2$ for the lower jaw $(\mathrm{mM}= \pm 0,02)$;

- the ratio of the sum of the mesio-distal sizes of three teeth of the frontal group to the width of the central incisor is $2,7 \pm 0,1$ for the upper jaw, and is $3,4 \pm 0,1$ for the lower jaw $(\mathrm{mM}= \pm 0,01)$;

- $\quad$ the ratio of the distance between the most prominent points of the Zy-Zy zygomatic arches to the width of the canine is $18 \pm 0,6$ for the upper jaw, and is $20,8 \pm 0,8$ for the lower jaw ( $\mathrm{mM}= \pm 0,08)$;

- the ratio of the distance between the most prominent points of the Zy-Zy zygomatic arches to the width of the lateral incisor is $20,4 \pm 0,7$ for the upper jaw, and is $23,1 \pm 0,7$ for the lower jaw $(\mathrm{mM}= \pm 0,08)$;

- the ratio of the distance between the most prominent points of the zygomatic arches of Zy-Zy to the width of the central incisor is $16,2 \pm 0,6$ for the upper jaw, and is $26,2 \pm 0,8$ for the lower jaw $(\mathrm{mM}= \pm 0,08)$.

In most cases, a guideline for choosing the size of artificial front teeth is the distance between the canine lines applied on the occlusal wax pattern, for a dental technician. To determine the canine line and apply it to the wax pattern, the doctor needs to raise the lip, while the wing of the nose changes its position. As a result of which the definition of the canine line relative to the wing of the nose does not correspond to the actual position of the canines [8] (see Fig. 1).
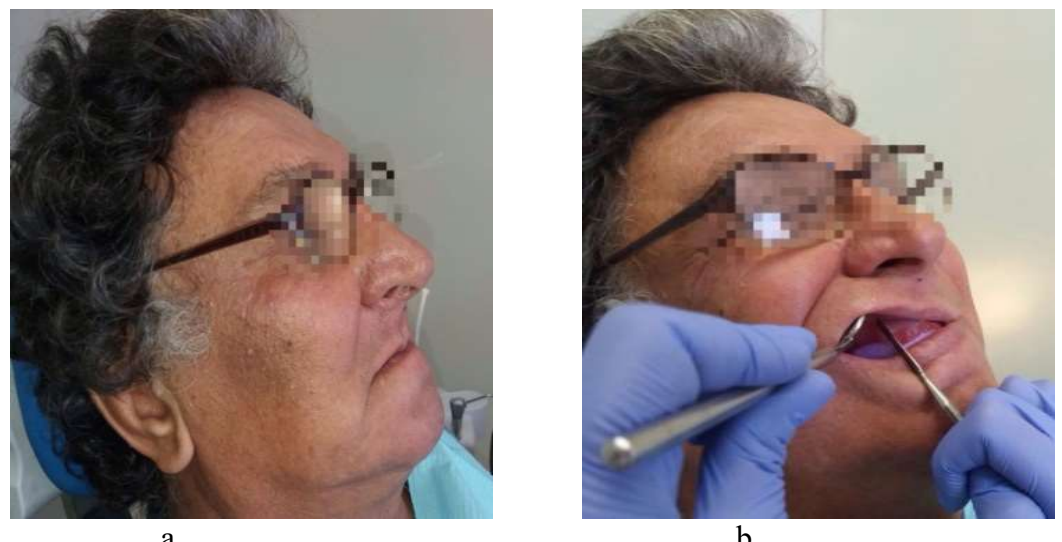

b

Fig. 1: (a) The Position of the Wings of the Nose at Rest; (b) Change in the Position of the Nose Wing When Applying Canine Line in the Wax Pattern.

Often for the same reason, the canine lines on the wax pattern can be at different distances from the midline, which reduces the quality of the prosthesis. An individual approach to the selection of the sizes of artificial front teeth will allow to solve the problems arising during the clinical consultation of the orthopedic dentist and the technical difficulties in choosing the size of artificial teeth during prosthetics. 
The example of the clinical case below shows the effectiveness of the application of the developed technique.

Clinical Case: Patient M., 65 years old, went to an orthopedic doctor for prosthetics. There was an experience with removable dentures in the anamnesis.

The patient complained of difficulty in nibbling, maceration of the corners of the mouth and aesthetic flaws, including the dark corners of the mouth when smiling in photographs. An external examination showed a deepening of the nasolabial and chin folds and a decrease in the height of the lower part of the face. A violation of the aesthetics of a smile was revealed. It was expressed in the omission of the corners of the mouth and was accompanied by wide cheek corridors. On examination of the oral cavity, it was established that removable laminar prostheses in the upper and lower jaws have atypical occlusive contacts in the lateral regions due to the narrowed upper dentition (Fig. 2).

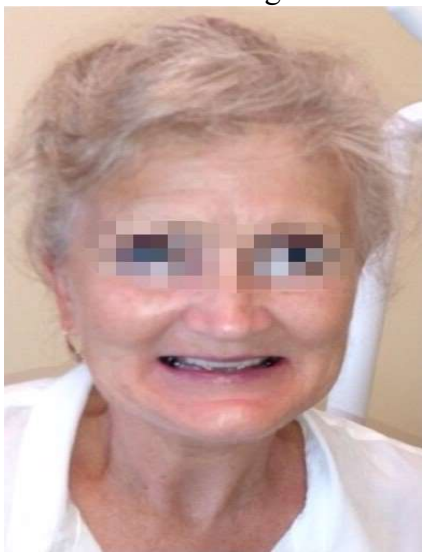

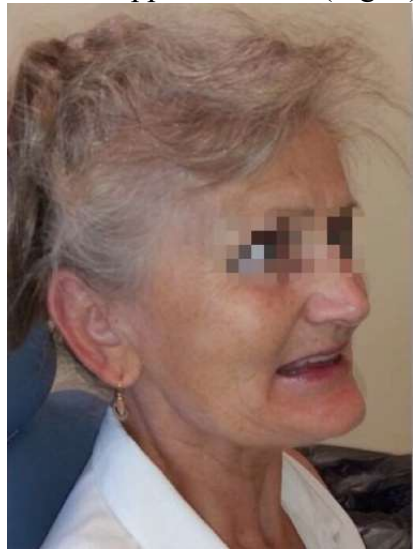

b

Fig. 2: (a) The Appearance of the Patient at the Time of Treatment (b) Wide Cheek Corridors and Lowering the Corners of the Mouth with a Smile.

Diagnosis: K08.1. Complete absence of teeth in the upper and lower jaw. I, II types of Oksman's classification. I types of mucous membrane of Supple's classification.

The treatment was carried out according to the clinical guidelines of the StAR. At the stage of prosthetic measures, the choice of the size of artificial teeth was carried out according to the method proposed by us. The parameters of the craniofacial complex were measured, as a result of which the distance between the Zy-Zy cheekbone points for this patient was $130 \mathrm{~mm}$. The sum of the mesio-distal dimensions of the six front teeth of the upper jaw $\left(\sum 3-3\right.$ u.j. $)$ was determined by dividing the interskus distance by a factor 3,0 (for the upper jaw, respectively). The resulting value is $\sum 3-3 \mathrm{u} . \mathrm{j} .=43,3$ мм. The next step - computation of the individual sizes of each of the front teeth in the upper jaw using the previously obtained coefficients. The calculations are presented in the table 1.

\begin{tabular}{|c|c|c|}
\hline Required parameters & Calculation formula & The result $(\mathrm{mm})$ \\
\hline$\sum 3-3$ u.j. & $\mathrm{Zy}-\mathrm{Zy} \div 3,0$ & 43,3 \\
\hline Central incisor width & $\sum 1,2,3$ u.j. $\div 2,7$ & 8 \\
\hline Side incisor width & $\sum 1,2,3$ u.j. $\div 3,4$ & 6,4 \\
\hline Fang width & $\sum 1,2,3$ u.j. $\div 3,0$ & 7,2 \\
\hline
\end{tabular}

Table 1: The Calculation of the Individual Sizes of Each of the Front Teeth of the Upper Jaw

Similar calculations are performed for the lower jaw (using the appropriate coefficients). The results are presented in table 2.

\begin{tabular}{|c|c|c|}
\hline Required parameters & Calculation formula & The result (mm) \\
\hline$\sum 3-31 . j$ & $Z y-Z y \div 3,85$ & 16,9 \\
\hline Central incisor width & $\sum 1,2,31 . \mathrm{j} . \div 3,4$ & 5 \\
\hline Side incisor width & $\sum 1,2,31 . \mathrm{j} . \div 3,0$ & 6,3 \\
\hline Fang width & $\sum 1,2,31 . \mathrm{j} . \div 2,7$ & 5,3 \\
\hline
\end{tabular}

Table 2: The Calculation of the Individual Sizes of Each of the Front Teeth of the Lower Jaw

Selection of artificial teeth was carried out by the dental technician on the individual sizes. The shape and height of the teeth was determined by the orthopedic doctor considering the facial parameters. As a result of the manufacture of new removable laminar dentures, an improvement in the patient's aesthetic parameters was achieved (Fig. 3). 


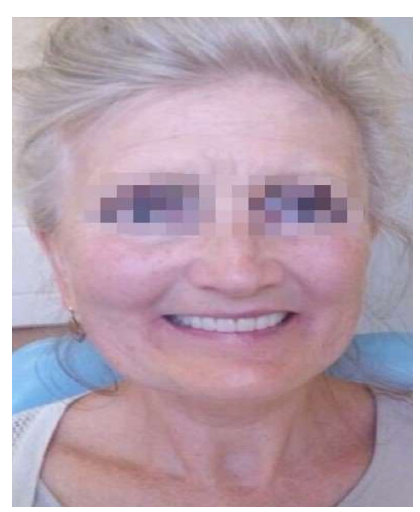

a

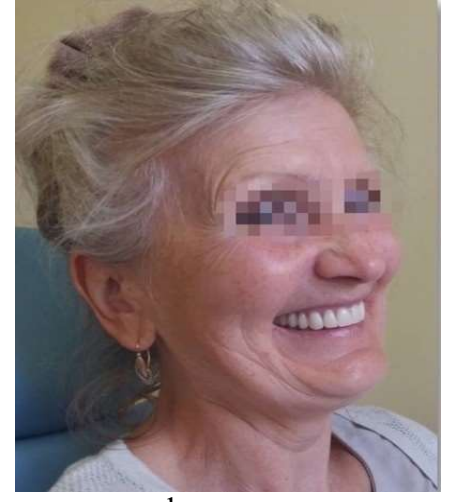

b

Fig. 3: Appearance of the Patient After Prosthetics According to the Proposed Method: (a) Lack of Cheek Corridors; (b) Corners of the Mouth are Raised, Nasolabial Folds Correspond to Age.

Practical Recommendations: Based on the study, we have developed tables for the express determination of the mesiodistal dimensions of the front teeth of the upper and lower jaw (Table 3; Table 4).

\begin{tabular}{|c|c|c|c|c|c|}
\hline $\begin{array}{c}\text { Mesio-distal size } \\
\begin{array}{c}\text { Zy-Zy distance } \\
\text { (in mm) }\end{array}\end{array}$ & Canine & Lateral incisor & Central incisor & $\begin{array}{c}\text { The sum of the } \\
\text { three front teeth }\end{array}$ & $\begin{array}{c}\text { The sum of the } \\
\text { six front teeth }\end{array}$ \\
\hline 124 & $6,9 \pm 0,14$ & Zy-Zy/20,4 & Zy-Zy/16,2 & Zy-Zy/6,0 & Zy-Zy/3,0 \\
\hline 125 & $6,9 \pm 0,26$ & $6,1 \pm 0,16$ & $7,7 \pm 0,34$ & $20,7 \pm 0,54$ & $41,3 \pm 1,8$ \\
\hline 126 & $7,0 \pm 0,22$ & $6,2 \pm 0,23$ & $7,7 \pm 0,36$ & $20,8 \pm 0,8$ & $41,7 \pm 1,6$ \\
\hline 127 & $7,1 \pm 0,23$ & $6,2 \pm 0,22$ & $7,8 \pm 0,33$ & $21,2 \pm 0,7$ & $42,0 \pm 1,14$ \\
\hline 128 & $7,1 \pm 0,2$ & $6,3 \pm 0,27$ & $7,9 \pm 0,3$ & $21,3 \pm 0,77$ & $42,7 \pm 1,54$ \\
\hline 129 & $7,2 \pm 0,2$ & $6,3 \pm 0,2$ & $8,0 \pm 0,32$ & $21,5 \pm 0,72$ & $43,0 \pm 1,44$ \\
\hline 130 & $7,2 \pm 0,25$ & $6,4 \pm 0,2$ & $8,0 \pm 0,31$ & $21,7 \pm 0,76$ & $43,3 \pm 1,52$ \\
\hline 131 & $7,3 \pm 0,34$ & $6,4 \pm 0,23$ & $8,1 \pm 0,37$ & $21,8 \pm 0,93$ & $43,7 \pm 1,86$ \\
\hline 132 & $7,3 \pm 0,3$ & $6,5 \pm 0,21$ & $8,1 \pm 0,34$ & $22,0 \pm 0,75$ & $44,0 \pm 1,5$ \\
\hline 133 & $7,4 \pm 0,2$ & $6,5 \pm 0,25$ & $8,2 \pm 0,3$ & $22,2 \pm 0,75$ & $44,3 \pm 1,5$ \\
\hline 134 & $7,4 \pm 0,21$ & $6,6 \pm 0,2$ & $8,3 \pm 0,3$ & $22,3 \pm 0,71$ & $44,7 \pm 1,42$ \\
\hline 135 & $7,5 \pm 0,2$ & $6,6 \pm 0,2$ & $8,3 \pm 0,33$ & $22,5 \pm 0,73$ & $45,0 \pm 1,46$ \\
\hline 136 & $7,6 \pm 0,34$ & $6,7 \pm 0,2$ & $8,4 \pm 0,3$ & $22,7 \pm 0,74$ & $45,3 \pm 1,48$ \\
\hline
\end{tabular}

Table 3: Determination of the Optimal Mesio-Distal Dimensions of the Front Teeth of the Upper Jaw, Depending on the Interscale Distance (mm)

\begin{tabular}{|c|c|c|c|c|c|}
\hline $\begin{array}{c}\text { Mesio-distal } \\
\text { size }\end{array}$ & Canine & Lateral incisor & Central incisor & $\begin{array}{c}\text { The sum of the } \\
\text { three front teeth }\end{array}$ & $\begin{array}{c}\text { The sum of the } \\
\text { six front teeth }\end{array}$ \\
\hline $\begin{array}{c}\text { Zy-Zy distance } \\
\text { (in mm) }\end{array}$ & $\mathrm{Zy}-\mathrm{Zy} / 20,8$ & $\mathrm{Zy}-\mathrm{Zy} / 23,1$ & $\mathrm{Zy}-\mathrm{Zy} / 26,2$ & $\mathrm{Zy}-\mathrm{Zy} / 7,7$ & $\mathrm{Zy}-\mathrm{Zy} / 3,85$ \\
\hline 124 & $6,0 \pm 0,23$ & $5,4 \pm 0,27$ & $4,7 \pm 0,3$ & $16,1 \pm 0,8$ & $32,2 \pm 1,6$ \\
\hline 125 & $6,0 \pm 0,17$ & $5,4 \pm 0,23$ & $4,8 \pm 0,3$ & $16,2 \pm 0,7$ & $32,5 \pm 1,4$ \\
\hline 126 & $6,1 \pm 0,21$ & $5,5 \pm 0,12$ & $4,8 \pm 0,32$ & $16,4 \pm 0,65$ & $32,7 \pm 1,3$ \\
\hline 127 & $6,1 \pm 0,2$ & $5,5 \pm 0,14$ & $4,8 \pm 0,3$ & $16,5 \pm 0,64$ & $33,0 \pm 1,28$ \\
\hline 128 & $6,2 \pm 0,2$ & $5,5 \pm 0,2$ & $4,9 \pm 0,34$ & $16,6 \pm 0,74$ & $33,2 \pm 1,48$ \\
\hline 129 & $6,2 \pm 0,25$ & $5,6 \pm 0,2$ & $4,9 \pm 0,29$ & $16,8 \pm 0,74$ & $33,5 \pm 1,48$ \\
\hline 130 & $6,3 \pm 0,23$ & $5,6 \pm 0,26$ & $5,0 \pm 0,33$ & $16,9 \pm 0,82$ & $33,8 \pm 1,64$ \\
\hline 131 & $6,3 \pm 0,16$ & $5,7 \pm 0,26$ & $5,0 \pm 0,34$ & $17,0 \pm 0,76$ & $34,0 \pm 1,52$ \\
\hline 132 & $6,3 \pm 0,27$ & $5,7 \pm 0,22$ & $5,0 \pm 0,37$ & $17,1 \pm 0,86$ & $34,3 \pm 1,72$ \\
\hline 133 & $6,4 \pm 0,21$ & $5,8 \pm 0,24$ & $5,1 \pm 0,43$ & $17,3 \pm 0,88$ & $34,5 \pm 1,76$ \\
\hline 134 & $6,4 \pm 0,15$ & $5,8 \pm 0,29$ & $5,1 \pm 0,38$ & $17,4 \pm 0,82$ & $34,8 \pm 1,74$ \\
\hline 135 & $6,5 \pm 0,2$ & $5,8 \pm 0,3$ & $5,2 \pm 0,41$ & $17,5 \pm 0,91$ & $35,1 \pm 1,82$ \\
\hline 136 & $6,5 \pm 0,2$ & $5,9 \pm 0,31$ & $5,2 \pm 0,39$ & $17,7 \pm 0,9$ & $35,3 \pm 1,8$ \\
\hline
\end{tabular}

Table 4: Determination of the Optimal Mesio-Distal Dimensions of the Front Teeth of the Lower Jaw, Depending on the Interscale Distance ( $\mathrm{mm})$ 


\section{Conclusion}

Broad prospects for the use of this method in dental practice are open up, and in particular, in prosthetics of patients with defects of dentition particularly in the anterior region, which must meet individual aesthetic parameters to a greater extent. Using the tables proposed by us, the dentist will be able to accurately predict the required size of artificial teeth. This reduces the time spent on the examination of the patient, and eliminates the error that is allowed when determining the canine line. In addition, the work of the dental technician at the choice of the necessary headset of artificial teeth is facilitated.

\section{References}

1. Ivanova O.P., Chernenko S.V. Individual approach to the selection of the size of the frontal group of artificial teeth. // Kazan: Modern Dentistry. Collection of scientific papers dedicated to the 125th anniversary of the founder of the Department of Orthopedic Dentistry of KSMU, Professor Isaak M. Oksman. - 2017 - pages. 173-177;

2. Chernenko S.V. Orthodontics of children and adults: Manual / S.V. Chernenko, P.A. Zhelezny, Yu.K. Zheleznaya, S.P. Zhelezny, O.P. Ivanova, O.S. Korchemnaya. - Moscow, LLC Mittel Press printing house, 2018. - 464 pages;

3. Lebedenko I. Yu., Kalivradzhiyan E. S., Ibragimov T. I. Guide to orthopedic dentistry. Prosthetics in the absence of teeth: Tutorial. - 3rd ed., Corrected. and add. //Moscow. - 2011. - 448 pages;

4. Modern Orthodontics / William R. Proffit; per. from English ; by ed. Corr. RAS, prof. L.S.Persina. - 4th ed. - M.: MEDPress-inform, 2017. - 560 pages;

5. Tanaka M. M., Johnston L. E. The prediction of the size of unerupted canines and premolars in a contemporary orthodontic population //The Journal of the American Dental Association. - 1974. - T. 88. - №. 4. - pages. 798-801;

6. Persin L.S. Orthodontics. Diagnosis and treatment of dentofacial anomalies and deformities: a textbook. - M.: GEOTAR-Media, 2016. - 640 pages;

7. Bolton W. A. The clinical application of a tooth-size analysis //American Journal of Orthodontics. - 1962. - T. 48. - №. 7. - pages. 504-529.

8. Kopeikin Century. N. Guide to orthopedic dentistry - M .: "Triad-X" 1998 - 373 pages.

9. Domenyuk D. A., Vedeshina E. G., Dmitrienko S. V. Correlation of the sizes of the teeth with the parameters of the maxillofacial arches and the maxillofacial area according to the results of the study of native skull preparations // Kuban Scientific Medical Journal. - 2016. - №. 2 